\title{
Notas Científicas \\ Respostas fisiológicas de tambaqui a banhos terapêuticos com mebendazol
}

\author{
Edsandra Campos Chagas ${ }^{(1)}$, Lucelle Dantas de Araújo(1) ${ }^{(1)}$ André Luiz Ferreira da Silva( ${ }^{(1)}$, \\ Levy de Carvalho Gomes $^{(1)}$ e Franmir Rodrigues Brandão(1)
}

(1)Embrapa Amazônia Ocidental, Caixa Postal 319, CEP 69011-970 Manaus, AM. E-mail: edsandra@cpaa.embrapa.br, levy@cpaa.embrapa.br

\begin{abstract}
Resumo - O objetivo deste trabalho foi avaliar as respostas fisiológicas de tambaqui (Colossoma macropomum, Cuvier 1818) a banhos terapêuticos com mebendazol. Juvenis de tambaqui ( $\mathrm{n}=108$, massa média de 37,80 $\pm 0,24 \mathrm{~g}$ ) foram submetidos a banhos terapêuticos de mebendazol a 0, 100, 300 e $600 \mathrm{mg} \mathrm{L}^{-1}$, com três repetições, em exposições de 30, 60 e 120 minutos. Não foram observadas diferenças significativas para íons plasmáticos (sódio, potássio e cálcio) e parâmetros hematológicos - hematócrito, número de eritrócitos e constantes corpusculares -, com exceção da hemoglobina, que apresentou aumento significativo, após 120 minutos na concentração de $300 \mathrm{mg} \mathrm{L}^{-1}$ de mebendazol, de forma similar ao que ocorreu com a glicose. Os indicadores fisiológicos de estresse avaliados revelam que o tambaqui mantém a sua homeostasia em concentrações de até $600 \mathrm{mg} \mathrm{L}^{-1}$ de mebendazol por até 120 minutos, com boa tolerância ao mebendazol.
\end{abstract}

Termos para indexação: Colossoma macropomum, estresse fisiológico, hematologia, concentração de íons plasmáticos, piscicultura.

\section{Physiological responses of tambaqui to therapeutic baths of mebendazole}

\begin{abstract}
The objective of this work was to evaluate the physiological responses of tambaqui (Colossoma macropomum, Cuvier 1818) to therapeutic baths with mebendazole. Tambaqui juveniles ( $\mathrm{n}=108$, mean weight $37.80 \pm 0.24 \mathrm{~g}$ ) were submitted to therapeutic baths with mebendazole at $0,100,300$ and $600 \mathrm{mg} \mathrm{L}^{-1}$, with three replicates, during 30, 60 and 120 minutes. No significant differences were observed for plasma ions (sodium, potassium and calcium) concentration and for hematological parameters (hematocrit, eritrocit numbers and corpuscular indices), except hemoglobin, which increase after exposure of 120 minutes in the mebendazole concentration of $300 \mathrm{mg} \mathrm{L}^{-1}$ was significant, similarly to the observed for glucose. Stress physiological indices evaluated showed that tambaqui maintains its homeostasis in concentrations until $600 \mathrm{mg} \mathrm{L}^{-1}$ of mebendazole for 120 minutes, presenting a good tolerance to mebendazole.
\end{abstract}

Index terms: Colossoma macropomum, physiological stress, hematology, plasma ions concentrations, fish culture.

O tambaqui, Colossoma macropomum (Cuvier 1818), espécie mais cultivada na Região Norte do Brasil (Val et al., 2000), apresenta bom desempenho em criação intensiva (Melo et al., 2001). Nesse sistema, os peixes são expostos continuamente a vários estressores (Barton \& Iwama, 1991; Wendelaar Bonga, 1997), como alterações na química da água, altas densidades de estocagem, manuseio excessivo e uso indiscriminado de drogas no tratamento de doenças (Wedemeyer, 1996).

As respostas ao estresse são divididas em três categorias: primária, secundária e terciária (Wendelaar
Bonga, 1997). Em geral, diante de um estressor, a resposta primária consiste na liberação de hormônios como o cortisol (Barton \& Iwama, 1991; Acerete et al., 2004); a secundária está relacionada ao metabolismo energético, como hiperglicemia e alteração da homeostase eletrolítica no sangue e tecidos (Barton \& Iwama, 1991; McDonald \& Milligan, 1997; Wendelaar Bonga, 1997); a terciária inclui o comprometimento no desempenho, mudanças no comportamento e aumento da suscetibilidade a doenças (Wedemeyer, 1996; Wendelaar Bonga, 1997). 
O tratamento de doenças parasitárias, como as causadas pelos monogenóides, parasitas de maior ocorrência em tambaquis criados em tanques-rede (Varella et al., 2003), ainda é um problema, principalmente porque os produtos usualmente aplicados são tóxicos para os peixes e para o meio ambiente. O controle de monogenóides tem sido realizado com o uso do mebendazol, bitionol e praziquantel, administrados por meio de banhos e oralmente (Schmahl \& Taraschewski, 1987; Kim \& Choi, 1998; Martins et al., 2001), sendo o mebendazol considerado uma das drogas mais eficazes e menos tóxicas para os peixes (Kim \& Choi, 1998; Martins et al., 2001). Contudo, não há relatos sobre tolerância do tambaqui às drogas utilizadas no controle de doenças parasitárias.

O objetivo deste trabalho foi avaliar as respostas fisiológicas de tambaqui (C. macropomum) expostos a banhos terapêuticos com mebendazol.

Juvenis de tambaqui (comprimento padrão, $13,48 \pm 0,48 \mathrm{~cm}$, e massa $37,80 \pm 0,24 \mathrm{~g}$ ) foram criados em tanques escavados de $200 \mathrm{~m}^{3}$ no setor de piscicultura da Embrapa Amazônia Ocidental e alimentados com ração comercial para peixes onívoros (36\% PB) até a saciedade aparente, seis dias por semana, durante o período pré-experimental.

Nos ensaios, 108 tambaquis foram distribuídos em 12 tanques de polietileno (100 L), segundo um delineamento inteiramente casualizado, em quatro tratamentos experimentais, correspondentes às concentrações de mebendazol de $0,100,300$ e $600 \mathrm{mg} \mathrm{L}^{-1}$, com três repetições por tratamento. Em cada unidade experimental, foram estocados nove animais, aclimatados por 24 horas antes do início dos banhos terapêuticos, que tiveram a duração de 120 minutos. Nesse período, a alimentação dos animais foi suspensa.

Parâmetros físico-químicos das unidades experimentais, como temperatura $\left(26,93 \pm 0,23^{\circ} \mathrm{C}\right)$, oxigênio dissolvido $\left(7,57 \pm 0,09 \mathrm{mg} \mathrm{L}^{-1}\right)$ e $\mathrm{pH}(6,81 \pm 0,05)$ foram monitorados durante a execução dos banhos terapêuticos.

A tolerância do tambaqui a banhos terapêuticos com mebendazol foi avaliada mediante análise de indicadores fisiológicos, em intervalos de 30, 60 e 120 minutos. Para isso, com os peixes previamente anestesiados (100 mg L $\mathrm{L}^{-1}$ de benzocaína), procedeu-se à coleta de sangue de nove peixes de cada tratamento (três de cada repetição) nos diferentes tempos de exposição, mediante punção de vasos caudais com seringas heparinizadas, para determinação do hematócrito (Ht), depois da centrifugação do sangue (12.000 g, 10 minutos), em tubos microcapilares heparinizados, e posterior leitura em escala padronizada; concentração de hemoglobina (Hb), segundo o método da cianometa-hemoglobina; e contagem do número de eritrócitos (RBC), realizada em uma câmara de Neubauer, depois da diluição do sangue em solução de formol citrato. Equações hematimétricas (Acerete et al., 2004) foram utilizadas para determinação das constantes corpusculares, como o volume corpuscular médio (VCM), a hemoglobina corpuscular média (HCM) e a concentração de hemoglobina corpuscular média (CHCM). A concentração de glicose foi determinada usando um monitor de glicose no sangue (Advantage). Depois desse procedimento, o sangue foi centrifugado a 3.500 rpm, por cinco minutos, para obtenção do plasma, visando à determinação dos níveis de $\mathrm{Na}^{+}$e $\mathrm{K}^{+}$, utilizando fotômetro de chama (B462, Micronal), e dos níveis de $\mathrm{Ca}^{2+}$, estimados por espectrofotometria de absorção atômica (Varian AA-1475).

Os resultados estão expressos em médiaterro-padrão da média. As diferenças entre as médias dos tratamentos foram estabelecidas por análise de variância de dois fatores (two way ANOVA) e as médias comparadas pelo teste de Tukey a 5\% de probabilidade.

Quanto às diferentes concentrações de mebendazol avaliadas, não foi observada a ocorrência de mortalidade de tambaquis em 30, 60 e 120 minutos de exposição. A avaliação hematológica não mostrou alterações significativas nos valores de Ht, RBC, VCM, HCM e CHCM nas diferentes concentrações de mebendazol e tempos de exposição (Tabela 1). Por sua vez, aumento significativo nos valores de Hb foi observado nos tambaquis expostos à concentração de $300 \mathrm{mg} \mathrm{L}^{-1}$ de mebendazol depois de 120 minutos, em comparação ao tempo de 60 minutos. Essa variação intra-específica na hemoglobina também foi observada por Tavares-Dias \& Mataqueiro (2004) em pacu (Piaractus mesopotamicus), em decorrência dos elevados valores do coeficiente de variação da hemoglobina, normalmente registrados em peixes sob uma mesma condição ambiental. Apesar dessa variação, relatada para hemoglobina, na concentração de $300 \mathrm{mg} \mathrm{L}^{-1}$ de mebendazol, os valores deste indicador e dos demais parâmetros hematológicos analisados foram similares aos verificados em tambaquis cultivados e outras 25 espécies de peixes da Bacia Amazônica (Marcon et al., 1999; Chagas et al., 2003), em pacu do Pantanal (Tavares-Dias \& Mataqueiro, 2004) e jundiá (Rhamdia quelen) (Borges et al., 2004). 
A concentração de glicose dos peixes expostos a $300 \mathrm{mg} \mathrm{L}^{-1}$ de mebendazol foi significativamente maior aos 120 minutos de exposição, se comparada a 30 e 60 minutos, e quando comparada aos valores obtidos nas concentrações 0 e $100 \mathrm{mg} \mathrm{L}^{-1}$ em 120 minutos de exposição (Tabela 1). Os níveis de sódio, potássio e cálcio plasmático não apresentaram diferenças significativas nas diferentes concentrações de mebendazol e tempos de exposição.

Tabela 1. Parâmetros fisiológicos de juvenis de tambaqui (Colossoma macropomum) após exposição ao mebendazol ${ }^{(1)}$.

\begin{tabular}{|c|c|c|c|c|}
\hline \multirow{2}{*}{$\begin{array}{l}\text { Tempo de } \\
\text { exposição } \\
\text { (minutos) }\end{array}$} & \multicolumn{4}{|c|}{ Mebendazol (mg L L ${ }^{-1}$} \\
\hline & 0 & 100 & 300 & 600 \\
\hline & \multicolumn{4}{|c|}{ Hematócrito (\%) } \\
\hline 30 & $31,97 \pm 1,69 \mathrm{a}$ & $30,45 \pm 1,04 \mathrm{a}$ & $30,69 \pm 1,30 \mathrm{a}$ & $28,33 \pm 1,69 a$ \\
\hline 60 & $33,28 \pm 1,92 \mathrm{a}$ & $34,53 \pm 1,79 a$ & $33,86 \pm 1,85 \mathrm{a}$ & $29,67 \pm 1,60 \mathrm{a}$ \\
\hline \multirow[t]{2}{*}{120} & $33,33 \pm 1,04 a$ & $32,50 \pm 2,50 \mathrm{a}$ & $33,11 \pm 0,62 a$ & $32,81 \pm 1,62 \mathrm{a}$ \\
\hline & \multicolumn{4}{|c|}{ Hemoglobina $\left(\mathrm{g} \mathrm{dL}^{-1}\right)$} \\
\hline 30 & $8,38 \pm 0,62 a$ & $8,41 \pm 0,52 \mathrm{a}$ & $9,38 \pm 0,79 \mathrm{ab}$ & $9,27 \pm 0,47 \mathrm{a}$ \\
\hline 60 & $10,01 \pm 0,98 \mathrm{a}$ & $10,16 \pm 0,34 \mathrm{a}$ & $8,52 \pm 0,67 \mathrm{a}$ & $8,61 \pm 0,29 a$ \\
\hline \multirow[t]{2}{*}{120} & $9,36 \pm 0,26 \mathrm{a}$ & $9,89 \pm 0,37 \mathrm{a}$ & $11,01 \pm 0,37 \mathrm{~b}$ & $9,86 \pm 0,46 \mathrm{a}$ \\
\hline & \multicolumn{4}{|c|}{ Número de eritrócitos $\left(10^{6} \mathrm{~mm}^{-3}\right)$} \\
\hline 30 & $2,51 \pm 0,15 \mathrm{a}$ & $2,26 \pm 0,26 \mathrm{a}$ & $2,24 \pm 0,26 \mathrm{a}$ & $2,17 \pm 0,25 \mathrm{a}$ \\
\hline 60 & $2,42 \pm 0,19 \mathrm{a}$ & $2,34 \pm 0,19 \mathrm{a}$ & $2,23 \pm 0,21 \mathrm{a}$ & $1,86 \pm 0,18 \mathrm{a}$ \\
\hline \multirow[t]{2}{*}{120} & $2,55 \pm 0,23 \mathrm{a}$ & $2,52 \pm 0,30 \mathrm{a}$ & $2,50 \pm 0,16 \mathrm{a}$ & $2,28 \pm 0,18 \mathrm{a}$ \\
\hline & \multicolumn{4}{|c|}{ Volume corpuscular médio $\left(\mu \mathrm{m}^{3}\right)$} \\
\hline 30 & $130,44 \pm 8,85 \mathrm{a}$ & $148,75 \pm 15,38 \mathrm{a}$ & $153,46 \pm 18,58 \mathrm{a}$ & $145,66 \pm 18,89 \mathrm{a}$ \\
\hline 60 & $140,08 \pm 6,79 a$ & $163,71 \pm 27,46 a$ & $160,30 \pm 13,74 a$ & $166,68 \pm 11,44 a$ \\
\hline \multirow[t]{2}{*}{120} & $143,48 \pm 11,02 \mathrm{a}$ & $144,30 \pm 16,88 \mathrm{a}$ & $154,85 \pm 11,24 \mathrm{a}$ & $150,55 \pm 12,91 \mathrm{a}$ \\
\hline & \multicolumn{4}{|c|}{ Hemoglobina corpuscular média (pg) } \\
\hline 30 & $34,21 \pm 2,94 a$ & $39,98 \pm 3,56 a$ & $47,25 \pm 6,31 \mathrm{a}$ & $47,79 \pm 6,63 a$ \\
\hline 60 & $41,33 \pm 2,95 \mathrm{a}$ & $47,26 \pm 6,41 \mathrm{a}$ & $39,39 \pm 2,81 \mathrm{a}$ & $48,79 \pm 3,55 \mathrm{a}$ \\
\hline \multirow[t]{2}{*}{120} & $38,72 \pm 2,99 \mathrm{a}$ & $43,76 \pm 4,88 \mathrm{a}$ & $45,12 \pm 2,59 \mathrm{a}$ & $45,04 \pm 3,68 \mathrm{a}$ \\
\hline & \multicolumn{4}{|c|}{ Concentração de hemoglobina corpuscular média (\%) } \\
\hline 30 & $26,49 \pm 2,00 \mathrm{a}$ & $27,66 \pm 1,47 \mathrm{a}$ & $30,56 \pm 2,21 \mathrm{a}$ & $33,06 \pm 1,44 a$ \\
\hline 60 & $29,59 \pm 1,71 \mathrm{a}$ & $29,91 \pm 1,51 \mathrm{a}$ & $25,29 \pm 1,69 a$ & $29,35 \pm 0,97 a$ \\
\hline \multirow[t]{2}{*}{120} & $27,08 \pm 1,01 \mathrm{a}$ & $31,60 \pm 2,00 \mathrm{a}$ & $29,66 \pm 1,39 \mathrm{a}$ & $30,36 \pm 1,42 \mathrm{a}$ \\
\hline & \multicolumn{4}{|c|}{ Glicose $\left(\mathrm{mg} \mathrm{dL}^{-1}\right)$} \\
\hline 30 & $58,80 \pm 3,03 \mathrm{a}$ & $55,56 \pm 3,54 a$ & $55,89 \pm 2,81 \mathrm{a}$ & $58,67 \pm 2,61 \mathrm{a}$ \\
\hline 60 & $58,63 \pm 3,14 \mathrm{a}$ & $52,86 \pm 2,71 \mathrm{a}$ & $52,67 \pm 1,76 a$ & $57,68 \pm 2,96 a$ \\
\hline \multirow[t]{2}{*}{120} & $58,11 \pm 2,63 \mathrm{aA}$ & $61,57 \pm 2,76 \mathrm{aA}$ & $73,13 \pm 3,01 \mathrm{bB}$ & $66,75 \pm 4,34 \mathrm{aAB}$ \\
\hline & \multicolumn{4}{|c|}{ Sódio plasmático $\left(\mathrm{mEq} \mathrm{L}^{-1}\right)$} \\
\hline 30 & $124,89 \pm 2,09 a$ & $126,25 \pm 2,75 a$ & $122,77 \pm 2,64 a$ & $128,18 \pm 1,53 a$ \\
\hline 60 & $121,99 \pm 2,92 \mathrm{a}$ & $127,02 \pm 2,25 \mathrm{a}$ & $118,90 \pm 5,91 \mathrm{a}$ & $122,55 \pm 4,85 \mathrm{a}$ \\
\hline \multirow[t]{2}{*}{120} & $119,31 \pm 3,68 \mathrm{a}$ & $126,05 \pm 2,30 \mathrm{a}$ & $123,76 \pm 1,75 a$ & $124,12 \pm 2,43 a$ \\
\hline & \multicolumn{4}{|c|}{ Potássio plasmático $\left(\mathrm{mEq} \mathrm{L}^{-1}\right)$} \\
\hline 30 & $5,55 \pm 0,29 \mathrm{a}$ & $5,80 \pm 0,14 \mathrm{a}$ & $5,70 \pm 0,35 \mathrm{a}$ & $6,06 \pm 0,15 \mathrm{a}$ \\
\hline 60 & $5,63 \pm 0,27 \mathrm{a}$ & $5,46 \pm 0,34 a$ & $5,70 \pm 0,34 a$ & $5,57 \pm 0,09 \mathrm{a}$ \\
\hline \multirow[t]{2}{*}{120} & $5,38 \pm 0,27 \mathrm{a}$ & $5,50 \pm 0,36 \mathrm{a}$ & $5,31 \pm 0,19 \mathrm{a}$ & $5,41 \pm 0,37 \mathrm{a}$ \\
\hline & \multicolumn{4}{|c|}{ Cálcio plasmático $\left(\mathrm{mEq} \mathrm{L}^{-1}\right)$} \\
\hline 30 & $5,84 \pm 0,16 \mathrm{a}$ & $6,08 \pm 0,30 \mathrm{a}$ & $6,12 \pm 0,16 \mathrm{a}$ & $5,93 \pm 0,14 a$ \\
\hline 60 & $5,90 \pm 0,21 \mathrm{a}$ & $6,24 \pm 0,16 a$ & $6,08 \pm 0,21 \mathrm{a}$ & $5,99 \pm 0,12 \mathrm{a}$ \\
\hline 120 & $5,84 \pm 0,11 \mathrm{a}$ & $5,91 \pm 0,23 \mathrm{a}$ & $5,94 \pm 0,16 \mathrm{a}$ & $6,48 \pm 0,13 a$ \\
\hline
\end{tabular}

(1)Médias seguidas por letras distintas, minúsculas nas colunas e maiúsculas nas linhas, apresentam diferença significativa em relação aos diferentes tempos de exposição e às diferentes concentrações de mebendazol, a 5\% de probabilidade pelo teste de Tukey.
A dosagem da glicose sangüínea é considerada um método valioso no diagnóstico da ocorrência de estresse fisiológico em peixes (Wendelaar Bonga, 1997; Acerete et al., 2004), por sua precisão e praticidade. A elevada concentração de glicose dos peixes expostos a $300 \mathrm{mg} \mathrm{L}^{-1}$ de mebendazol, em 120 minutos, pode estar relacionada à resposta individual, visto que diversos fatores ambientais e não ambientais influem nos níveis de glicose em peixes (Barton \& Iwama, 1991; TavaresDias \& Mataqueiro, 2004). Nesse estudo, em concentrações superiores (600 $\mathrm{mg} \mathrm{L}^{-1}$ de mebendazol), o tambaqui não apresentou elevação dos teores de glicose que caracterizasse a ativação de respostas de adaptação geral. Os indicadores fisiológicos avaliados mostram que o tambaqui, quando exposto ao mebendazol $(0,100$, 300 e $600 \mathrm{mg} \mathrm{L}^{-1}$ ), por até 120 minutos, ainda consegue manter a sua homeostasia.

O mebendazol tem sido empregado no controle de monogenóides, nos peixes, em concentrações de até $500 \mathrm{mg} \mathrm{L}^{-1}$, em banhos de curta duração (Martins et al., 2001). Neste estudo, o tambaqui apresentou boa tolerância ao mebendazol até $600 \mathrm{mg} \mathrm{L}^{-1}$, corroborando estudos realizados com outras espécies, que relatam que o mebendazol é uma droga com baixa toxicidade para os peixes (Kim \& Choi, 1998; Martins et al., 2001). Contudo, é necessário avaliar a eficácia dos banhos terapêuticos com mebendazol, mediante a realização da avaliação parasitológica dos animais para, com os resultados deste estudo, estabelecer uma concentração que apresente baixa toxicidade para peixes e alta eficácia no controle de monogenóides.

\section{Referências}

ACERETE, L.; BALASCH, J.C.; ESPINOSA, E.; JOSA, A.; TORT, L. Physiological responses in Eurasian perch (Perca fluviatilis, L.) subjected to stress by transport and handling. Aquaculture, v.237, p.167-178, 2004.

BARTON, B.A.; IWAMA, G.K. Physiological changes in fish from stress in aquaculture with emphasis on the response and effects of corticosteroids. Annual Review of Fish Diseases, v.1, p.3-26, 1991.

BORGES, A.; SCOTTI, L.V.; SIQUEIRA, D.R.; JURINITZ, D.F.; WASSERMANN, G.F. Hematologic and serum biochemical values for jundiá (Rhamdia quelen). Fish Physiology and Biochemistry, v.30, p.21-25, 2004.

CHAGAS, E.C.; LOURENÇO, J.N.P.; GOMES, L.C.; VAL, A.L. Desempenho e estado de saúde de tambaquis cultivados em tanquesrede sob diferentes densidades de estocagem. In: SIMPÓSIO BRASILEIRO DE AQÜICULTURA, 12., 2003, Goiânia. Anais. Jaboticabal: Aquabio, 2003. v.2, p.83-93. Editado por E.C. Urbinati, J.E.P. Cyrino. 
KIM, K.H.; CHOI, E.S. Treatment of Microcotyle sebastis (Monogenea) on the gills of cultured rockfish (Sebastes schelegeli) with oral administration of mebendazole and bithionol. Aquaculture, v.167, p.115-121, 1998.

MARCON, J.L.; CHAGAS, E.C.; KAVASSAKI, J.M.; VAL, A.L. Intra-erythrocytic phosphates in 25 fish species of the Amazon: GTP as a key factor in the regulation of $\mathrm{Hb}^{-\mathrm{O}_{2}}$ affinity. In: VAL, A.L.; VAL, V.M.F. de A. (Ed.). Biology of tropical fishes. Manaus: Inpa, 1999. p.229-240.

MARTINS, M.L.; ONAKA, E.M.; MORAES, F.R.; FUJIMOTO, R.Y. Mebendazole treatment against Anacanthorus penilabiatus (Monogenea, Dactylogyridae) gill parasite of cultivated Piaractus mesopotamicus (Osteichthyes, Characidae) in Brazil. Efficacy and hematology. Acta Parasitologica, v.46, p.332-336, 2001.

McDONALD, D.G.; MILLIGAN, C.L. Ionic, osmotic and acidbase regulation in stress. In: IWAMA, G.K.; PICKERING, A.D.; SUMPTER, J.P.; SCHRECK, C.B. (Ed.). Fish stress and health in aquaculture. Cambridge: Cambridge University Press, 1997. p.119-144.

MELO, L.A.S.; IZEL, A.C.U.; RODRIGUES, F.M. Criação de tambaqui (Colossoma macropomum) em viveiros de argila/ barragens no Estado do Amazonas. Embrapa Amazônia Ocidental, 2001. 30p. (Embrapa Amazônia Ocidental. Documentos, 18).

SCHMAHL, G.; TARASCHEWSKI, H. Treatment of fish parasites. 2. Effects of praziquantel, niclosamide, levamisole- $\mathrm{HCl}$, and metrifonate on monogenea (Gyrodactylus aculeati, Diplozoon paradoxum). Parasitology Research, v.73, p.341-351, 1987.

TAVARES-DIAS, M.; MATAQUEIRO, M.I. Características hematológicas, bioquímicas e biométricas de Piaractus mesopotamicus Holmberg, 1887 (Osteichthyes: Characidae) oriundos de cultivo intensivo. Acta Scientiarum, Biological Sciences, v.26, p.157-162, 2004.

VAL, A.L.; ROLIM, P.R.; RABELO, H. Situação atual da aqüicultura na Região Norte. In: VALENTI, W.C.; POLI, C.R.; PEREIRA, J.A.; BORGHETTI, J.R. (Ed.). Aqüicultura no Brasil: bases para um desenvolvimento sustentável. Brasília: CNPq/MCT, 2000. p.247266.

VARELLA, A.M.B.; PEIRO, S.N.; MALTA, J.C. de O.; LOURENÇO, J.N.P. Monitoramento da parasitofauna de Colossoma macropomum (Cuvier, 1818) (Osteichthyes: Characidae) cultivado em tanques-rede em um lago de várzea da Amazônia, Brasil. In: SIMPÓSIO BRASILEIRO DE AQÜICULTURA, 12., 2003, Goiânia. Anais. Jaboticabal: Aquabio, 2003. v.1, p.95-106. Editado por E.C. Urbinati, J.E.P. Cyrino.

WEDEMEYER, G.A. Physiology of fish in intensive culture systems. New York: Chapman \& Hall, 1996. 232p.

WENDELAAR BONGA, S.E. The stress response in fish. Physiological Reviews, v.77, p.591-625, 1997.

Recebido em 2 de maio de 2005 e aprovado em 4 de outubro de 2005 